\title{
Salmonella typhi Vi conjugate vaccine reduced the incidence of typhoid fever in 2 to 5 year old children
}

Lin FY, Ho VA, Khiem HB, et al. The efficacy of a Salmonella typhi Vi conjugate vaccine in two- to-five-year-old children. N Engl J Med 2001 Apr 26;344:1263-9.

\section{QUESTION: In Vietnamese children who are 2 to 5 years old, does vaccination with Salmonella typhi Vi conjugate vaccine reduce the incidence of typhoid fever?}

\section{Design}

Randomised (allocation concealed*), blinded (participants and outcome assessors),* placebo controlled trial with 27 months of follow up.

\section{Setting}

16 communes in Dong Thap Province, Vietnam.

\section{Participants}

12008 children who were 2 to 5 years of age (51\% boys). Children with illness that required ongoing medical care were excluded. $92 \%$ of children received 2 correctly labelled injections and completed the study.

\section{Intervention}

5991 children were allocated to $S$ typhi Vi conjugate vaccine (2 doses of $22.5 \mu \mathrm{g}$ of capsular polysaccharide and $22 \mu \mathrm{g}$ of Pseudomonas aeruginosa exotoxin A in $0.5 \mathrm{ml}$ of phosphate-buffered saline containing $0.01 \%$ thimerosal), and 6017 children were allocated to placebo. The children received 2 injections of either placebo or vaccine in the left deltoid muscle 6 weeks (range 28 to $57 \mathrm{~d}$ ) apart.

\section{Main outcome measures}

Incidence of blood culture, confirmed typhoid fever at 27 months, change in anti-S typhi Vi IgG antibodies over time, and adverse reactions.

\section{Main results}

At 27 months, more children were diagnosed with typhoid fever in the placebo group than in the vaccinated group (table). Overall, antibody concentrations increased by a factor of $>575(\mathrm{p}<0.001)$ in blood samples taken before the first injection and 4 weeks after the second. The antibody response persisted throughout the 2 year study period. No serious adverse reactions were reported.

\section{Conclusion}

In Vietnamese children who were 2 to 5 years old, the Salmonella typhi $\mathrm{Vi}$ conjugate vaccine reduced the incidence of typhoid fever more than placebo.

*See glossary.
Sources of funding: in part, National Institute of Child Health and Human Development and Aventis Pasteur.

For correspondence: Dr FY Lin, National Institute of Child Health and Human Development, Room 7B03, 6100 Executive Boulevard, Bethesda, MD 20892-7510, USA.Fax +1301402 2084.

Incidence of typhoid fever at 27 months for Salmonella typhi Vi conjugate vaccine v placebo in Vietnamese childrent

\begin{tabular}{lllll} 
Protocol & Vaccine & Placebo & RRR (95\% CI) & NNT (Cl) \\
All randomly allocated children & $0.08 \%$ & $0.93 \%$ & $91 \%(78$ to 96$)$ & $119(89$ to 163$)$ \\
\hline Children who received 2 correctly labelled injections & $0.07 \%$ & $0.84 \%$ & $91 \%(77$ to 97$)$ & $130(95$ to 184$)$ \\
\hline
\end{tabular}

†Abbreviations defined in glossary; RRR, NNT, and $\mathrm{Cl}$ calculated from data in article.

\section{COMMENTARY}

Typhoid fever continues to be a major health problem in developing countries, as well as a concern to travellers. Although effective typhoid vaccines have been used for almost 100 years, vaccination against typhoid has, to date, played a limited preventive role. 3 vaccines are available, but each has limitations. ${ }^{1}$

The results of the protein-conjugated Vi vaccine trial in Vietnam by Lin $e t$ al are exciting. Conjugate vaccines elicit T-dependent immune responses, are more immunogenic, and are more effective in young children. In this trial, 2 doses of Vi conjugate vaccine were $91 \%$ effective (95\% CI 78\% to $96 \%$ ) in preventing culture-proven typhoid among children 2 to 5 years of age in a highly endemic region. Among school-aged children and adults, the efficacy of the new vaccine will probably be at least as high as that among the young children.

Several important questions remain unanswered about this vaccine. How long will protection last? Will it, like other conjugate vaccines, be effective in infants, allowing it to be incorporated into existing childhood immunisation programmes? School-aged children have the greatest burden of typhoid, but morbidity is also high among pre-school children. School-based typhoid vaccination programmes are feasible and have been shown to decrease community attack rates, ${ }^{2}$ but they require a new infrastructure and do not protect younger children. How might this vaccine be best used? Travellers to endemic areas, military personnel, and residents of highly endemic countries are likely candidates. Novel uses for a highly effective vaccine should be considered, including aiding in the control of epidemics that result from highly resistant organisms. ${ }^{3}$

Andrew T Pavia, MD University of Utah Health Sciences Center Salt Lake City, Utah, USA

1 Woodruff BA, Pavia AT, Blake PA. A new look at typhoid vaccination. Information for the practicing physician. JAMA 1991;265:756-9.

2 Levine MM, Ferreccio C, Black RE, Tacket CO, Germanier R. Progress in vaccines against typhoid fever. Rev Infect Dis 1989;11(Suppl 3):S552-67.

3 Taylor DN, Levine MM, Kuppens L, Ivanoff B. Why are typhoid vaccines not recommended for epidemic typhoid fever? J Infect Dis 1999;180:2089-90. 